expression group (figure 3,4). The results showed that patients with high HOXC8 expression had a worse prognosis, suggesting that HOXC8 is an oncogene (figure 5). The results of GSEA analysis showed that high HOXC8 expression was

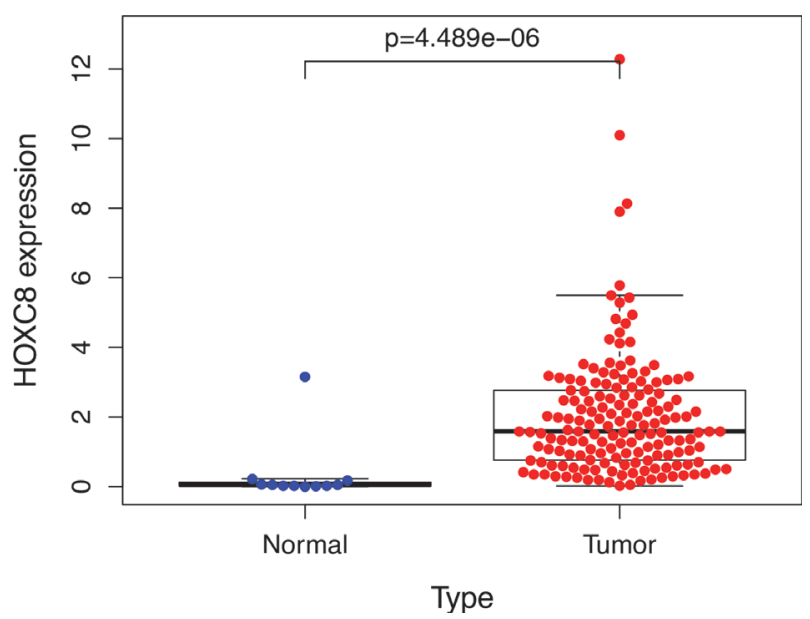

Abstract IDDF2020-ABS-0037 Figure 3

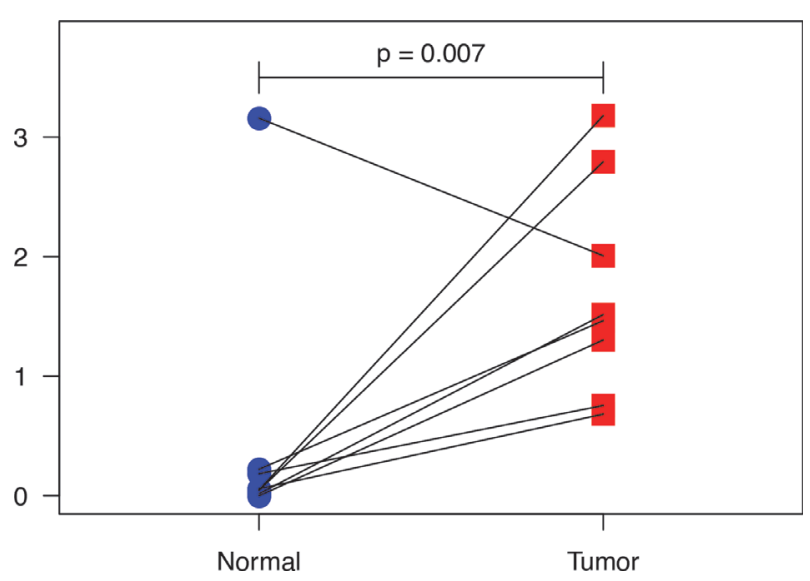

Abstract IDDF2020-ABS-0037 Figure 4

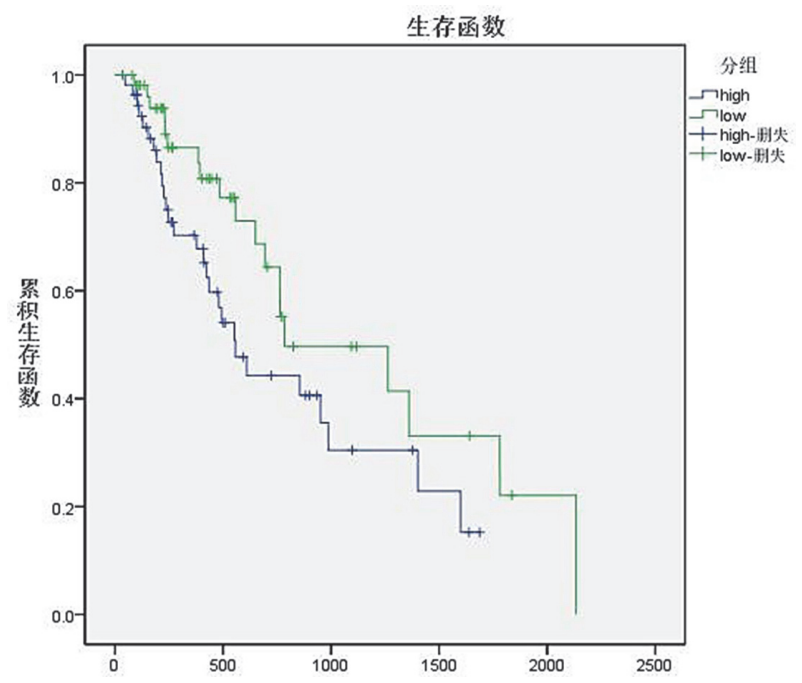

Abstract IDDF2020-ABS-0037 Figure 5

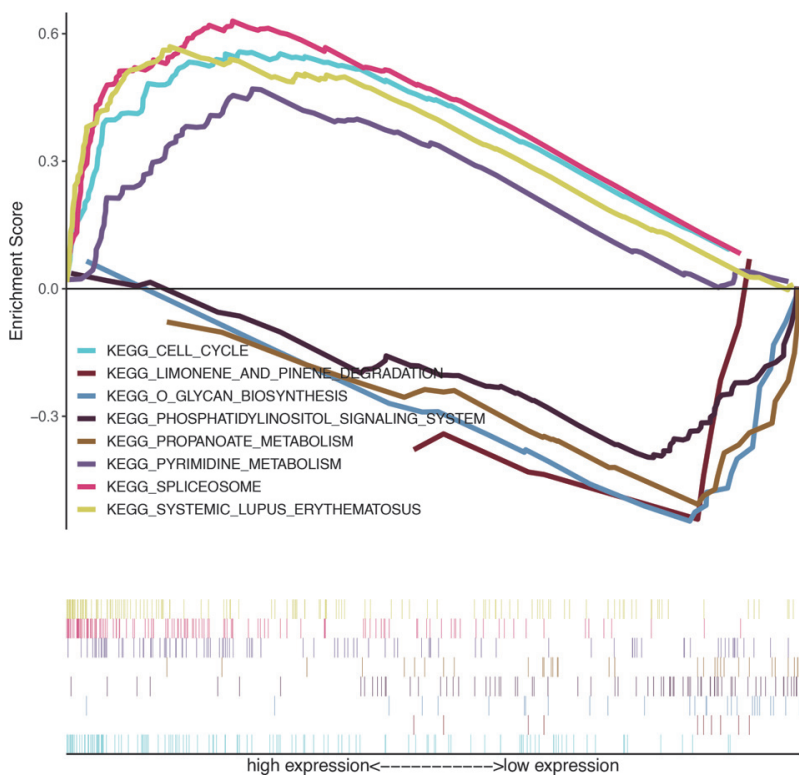

Abstract IDDF2020-ABS-0037 Figure 6

enriched in the cell cycle, splicing and other related gene sets, while low HOXC8 expression was enriched in phosphatidylinositol signaling pathway and other related gene sets (figure 6).

Conclusions HOXC8 may be one of the biomarkers for early diagnosis and prognosis of esophageal cancer. This will further study the role of HOXC8 in esophageal cancer in the future. The mechanism provides the basis and direction.

\section{IDDF2020-ABS-0046 HOXC6 PROMOTES METASTASIS BY RECRUITING MACROPHAGES AND ORCHESTRATING THE IL6/HOXC6/DKK1/ WNT/B-CATENIN AXIS IN RIGHT-SIDED COLON CANCER}

Lina Qi* Biting Zhou, Jiani Chen, Wangxiong Hu. Zhejiang University, China

\subsection{6/gutjnl-2020-IDDF.19}

Background Patients with right-sided colon cancer (RCC) generally have a poorer prognosis than those with left-sided colon cancer (LCC). We previously found that homeobox C6 (HOXC6) was the most significantly upregulated gene in RCC compared to LCC. However, it remains unclear whether HOXC6 plays a role in tumor proliferation and metastasis. Our study aimed to explore the potential oncogenic role and the detailed molecular mechanism of HOXC6 in RCC.

Methods Expression datasets from The Cancer Genome Atlas (TCGA) and Gene Expression Omnibus (GEO) databases were used to uncover the underlying oncogenic role of HOXC6 in RCC. CCK8, transwell, subcutaneous tumor cell injection and tail vein tumor cell injection assays were used to evaluate the proliferation, migration, and invasion of tumor cells in vitro and in vivo. Immunohistochemistry and immunofluorescence were performed to evaluate the correlation of HOXC6 and M2 macrophage infiltration. Western blot, qRT-PCR, ELISA, co-IP, and luciferase reporter assays were performed to examine the association of HOXC6 with epithelial-mesenchymal transition (EMT). 
Results HOXC6 was overexpressed in RCC and associated with poor prognosis. Overexpression of HOXC6 promoted the migration and invasion of CRC cells in vitro and in vivo. Increase in CCL2 expression by upregulation of HOXC6 could attract more infiltrating M2 macrophages. IL6 secreted by M2 macrophages could induce EMT in tumor cells by upregulating HOXC6 and activating the Wnt/ $\beta$-catenin signaling pathway via inhibition of DKK1 secretion.

Conclusions Our study indicates that overexpression of HOXC6 induces EMT by regulating the DKK1/Wnt/ $\beta$-catenin axis. The positive crosstalk between M2 macrophages and HOXC6 in tumors led to poor prognosis of RCC.

\section{IDDF2020-ABS-0090 MALONDIALDEHYDE LEVELS IN THE TESTICULAR ORGAN OF HYPERLIPIDEMIC RAT (RATTUS NORVEGICUS) WITH QUAIL EGG YOLK DIET}

'Muhammad Luthfi Adnan*, ${ }^{1}$ Dini Islamiana, ${ }^{1}$ Hilmi Ardian Sudarto, ${ }^{2}$ Miranti Dewi Pramaningtyas. 'Undergraduate Program of Medicine, Faculty of Medicine, Universitas Islam Indonesia, Indonesia; ${ }^{2}$ Departement of Physiology, Faculty of Medicine, Universitas Islam Indonesia, Indonesia

\subsection{6/gutjin-2020-IDDF.20}

Background Hyperlipidemia can cause infertility due to cell damage to the testicular organs. Hyperlipidemia can induce lipid peroxidation, which results in the formation of malondialdehyde (MDA). Quail egg yolk is one of the many foods consumed by Asians that contain high lipid levels. The aim of this study was to determine the effect of quail egg yolk diet on MDA levels of testicular organ on the rat.

Methods The subjects are male Wistar (Rattus norvegicus) strain rats 2-3 months with body weight 200-300 grams divided into two groups ( $\mathrm{K}+$ and $\mathrm{K}-)$. Group of $\mathrm{K}+$ were given quail egg yolk for two weeks with a dose of $5 \mathrm{ml}$ while group of K- were only given fed ad libitum. All rats terminated to taken the testicular organ to measure the level of MDA. All data were statistically analyzed with one-way ANOVA. Values were considered significant at $\mathrm{p}<0,05$.

Results Mean of MDA level (nmol/gram) in rats was 0,95 \pm 0,75 in $\mathrm{K}$ - group and $8,64 \pm 0,13$ in $\mathrm{K}+$ group. The oneway ANOVA test showed significant differences in activity between the group with $\mathrm{p}<0,001$ and Post Hoc test $\mathrm{p}<0,001$. Conclusions Quail egg yolk significantly increases MDA Levels in the testicular organ of hyperlipidemic rats. Consumption of quail egg yolks can affect the cell activity of testicular organs.

\section{IDDF2020-ABS-0100 LONG INTERGENIC NON-PROTEIN CODING RNA 01446 FACILITATES THE PROLIFERATION AND METASTASIS OF GASTRIC CANCER CELLS THROUGH INTERACTING WITH THE HISTONE LYSINE- SPECIFIC DEMETHYLASE LSD1}

${ }^{1}$ Weibin Lian*, ${ }^{2}$ Hongzhi Xu, ${ }^{2}$ Jianlin Ren, ${ }^{2}$ Changsheng Yan, ${ }^{2}$ Yifan Lian. ${ }^{1}$ Quanzhou First Hospital Affiliated to Fujian Medical University, China; ${ }^{2}$ Zhongshan Hospital, Xiamen University, China

\subsection{6/gutjnl-2020-IDDF.2}

Background Growing evidence illustrated that long non-coding RNAs (lncRNAs) exhibited widespread effects on the progression of human cancers via various mechanisms. Long intergenic non-protein coding RNA 01446 (LINC01446), a 3484 bp ncRNA, is known to locate at chromosome $7 \mathrm{p} 12.1$. However, its biological functions and specific action mechanism in gastric cancer (GC) are still unclear.

Methods LINC01446 expression levels in GC cells were detected by quantitative real-time PCR (qRT-PCR). LINC01446 siRNAs and overexpression vector were transfected into GC cells to down-regulate or up-regulate LINC00460 expression. The biological functions of LINC01446 were investigated in vitro and in vivo. Transcriptome RNA-seq, Western blot, RIP, RNA pull-down and ChIP assays were used to determine the mechanism of LINC01446 in regulating underlying targets.

Results In our study, LINC01446 was proved to be markedly up-regulated in GC tissues relative to and positively correlated with the poor survival of GC patients. The multivariate Cox regression model showed that LINC01446 functioned as an independent prognostic factor for the survival of GC patients. Functionally, LINC01446 facilitated the proliferation and metastasis of GC cells. Moreover, RNA-seq analysis demonstrated that LINC01446 knockdown primarily regulated the genes relating to the growth and migration of GC. Mechanistically, LINC01446 could widely interact with histone lysinespecific demethylase LSD1 and recruit LSD1 to the Ras-related dexamethasone-induced 1 (RASD1) promoter, thereby suppressing RASD1 transcription.

Conclusions Overall, these findings suggest that LINC01446/ LSD1/RASD1 regulatory axis may provide bona fide targets for anti-GC therapies.

\section{IDDF2020-ABS-0112 GUT-SKIN AXIS: DECODING THE LINK BETWEEN THE GUT MICROBIOME AND HIVES}

Learn-Han Lee*, Vengadesh Letchumanan, Loh Teng-Hern Tan, Hooi-Leng Ser, Jodi WoanFei Law. Novel Bacteria and Drug Discovery Research Group (NBDD), Microbiome and Bioresource Research Strength (MBRS), Jeffrey Cheah School of Medicine and Health Sciences, Monash University Malaysia, Malaysia

\subsection{6/gutjnl-2020-IDDF.22}

Background Urticaria (hives) is a rash affecting the skin and mucosa, often characterized by appearance wheals, angioedema, and pruritus. Chronic urticaria (CU) is classified by the occurrence of urticaria which exceeded six weeks (almost daily) without specific triggers and identifiable cause. CU is a common disease that has detrimental effects on quality of life. However, its aetiology remains unclear. There is increasing evidence that dysbiosis of the intestinal microbiota is associated with dermatologic conditions. The human gut microbiome has a significant role in the regulation of the immune system, which can be implicated in the development of immune-mediated diseases such as CU. This systematic review aims to investigate the relationship of gut bacteria and the development of CU.

Methods The systematic literature search was executed in accordance with the Preferred Reporting Items for Systematic Reviews and Meta-Analyses (PRISMA) guideline. Research commenced using $\mathrm{MeSH}$ terms relevant to the topic, ('gut' OR 'microbiome' OR 'microbiota' OR 'microflora') AND ('urticaria' OR 'hives') were performed on four databases (PubMed, EMBASE, ProQuest, Scopus; publication date limit to 29 February 2020). Titles and abstracts of all retrieved 